J. Nonlinear Var. Anal. 3 (2019), No. 3, pp. 257-275

Available online at http://jnva.biemdas.com

https://doi.org/10.23952/jnva.3.2019.3.03

\title{
EXPANDING THE APPLICABILITY OF AN ITERATIVE REGULARIZATION METHOD FOR ILL-POSED PROBLEMS
}

\author{
IOANNIS K. ARGYROS ${ }^{1}$, SANTHOSH GEORGE ${ }^{2, *}$ \\ ${ }^{1}$ Department of Mathematicsal Sciences, Cameron University, Lawton, OK 73505, USA \\ ${ }^{2}$ Department of Mathematical and Computational Sciences, \\ National Institute of Technology Karnataka, Mangaluru 575 025, India
}

\begin{abstract}
An iteratively regularized projection method, which converges quadratically, is considered for stable approximate solutions to a nonlinear ill-posed operator equation $F(x)=y$, where $F: D(F) \subseteq X \rightarrow X$ is a nonlinear monotone operator defined on the real Hilbert space $X$. We assume that only a noisy data $y^{\delta}$ with $\left\|y-y^{\delta}\right\| \leq \delta$ are available. Under the assumption that the Fréchet derivative $F^{\prime}$ of $F$ is Lipschitz continuous, a choice of the regularization parameter using an adaptive selection of the parameter and a stopping rule for the iteration index using a majorizing sequence are presented. We prove that, under a general source condition on $x_{0}-\hat{x}$, the error $\left\|x_{n, \alpha}^{h, \delta}-\hat{x}\right\|$ between the regularized approximation $x_{n, \alpha}^{h, \delta},\left(x_{0, \alpha}^{h, \delta}:=P_{h} x_{0}\right.$, where $P_{h}$ is an orthogonal projection on to a finite dimensional subspace $X_{h}$ of $X$ ) and the solution $\hat{x}$ is of optimal order.

Keywords. Majorizing sequence; Monotone operator; Nonlinear ill-posed operator; Quadratic convergence; Regularized Projection method.
\end{abstract}

2010 Mathematics Subject Classification. 65J20, 65J15, 47J06.

\section{INTRODUCTION}

Let $X$ be a real Hilbert space. Let $F: D(F) \rightarrow X$ with domain $D(F) \subseteq X$ be a monotone operator. We consider the problem of solving the nonlinear ill-posed operator equation

$$
F(x)=y
$$

approximately when the data $y$ is not known exactly. Assume that $y^{\delta} \in X$ are the available noisy data with

$$
\left\|y-y^{\delta}\right\| \leq \delta
$$

and that (1.1) has a solution $\hat{x}$. Equation (1.1) is ill-posed in the sense that the Fréchet derivative $F^{\prime}($.$) is$ not boundedly invertible (see, [19, page 26]). Since (1.1) is ill-posed, one has to replace equation (1.1) by a nearby equation whose solution is less sensitive to perturbation in the right side $y$. This replacement is known as regularization. A well known method for regularizing (1.1), when $F$ is monotone, is the method of the Lavrentiev regularization (see, [20]). In this method, approximation $x_{\alpha}^{\delta}$ is obtained by solving the singularly perturbed operator equation

$$
F(x)+\alpha\left(x-x_{0}\right)=y^{\delta} .
$$

${ }^{*}$ Corresponding author.

E-mail addresses: ioannisa@ cameron.edu (I. K. Argyros), sgeorge@nitk.edu.in; nitksanthosh@gmail.com (S. George).

Received July 3, 2017; Accepted May 4, 2019.

(c)2019 Journal of Nonlinear and Variational Analysis 
In practice, one has to deal with some sequence $\left(x_{n, \alpha}^{\delta}\right)$ converging to $\hat{x}$, the solution of (1.1). Recently, many authors considered such sequences; see $[6,7,8,12,17,18]$ and the references therein.

In [6], Bakushinsky and Smirnova considered an iteratively regularized Lavrentiev method:

$$
x_{k+1}^{\delta}=x_{k}^{\delta}-\left(A_{k}^{\delta}+\alpha_{k} I\right)^{-1}\left(F\left(x_{k}^{\delta}\right)-y^{\delta}+\alpha_{k}\left(x_{k}^{\delta}-x_{0}\right)\right),
$$

for $k=0,1,2, \cdots$, where $A_{k}^{\delta}:=F^{\prime}\left(x_{k}^{\delta}\right)$ and $\left(\alpha_{k}\right)$ is a sequence of positive real numbers such that $\lim _{k \rightarrow \infty} \alpha_{k}=0$ as an approximate solution for (1.1). A general discrepancy principle was considered in [6] for choosing the stopping index $k_{\delta}$ and showed that $x_{k_{\delta}}^{\delta} \rightarrow \hat{x}$ as $\delta \rightarrow 0$. However, no error estimate for $\left\|x_{k_{\delta}}^{\delta}-\hat{x}\right\|$ was given in [6]. Later, Mahale and Nair [13] considered method (1.4) and obtained an error estimate for $\left\|x_{k_{\delta}}^{\delta}-\hat{x}\right\|$ under weaker assumptions than the assumptions in [6].

In [9], George and Elmahdy considered the iterative regularization method

$$
x_{n+1, \alpha}^{\delta}=x_{n, \alpha}^{\delta}-\left(F^{\prime}\left(x_{0}\right)+\alpha I\right)^{-1}\left(F\left(x_{n, \alpha}^{\delta}\right)-y^{\delta}+\alpha\left(x_{n, \alpha}^{\delta}-x_{0}\right)\right),
$$

where $x_{0, \alpha}^{\delta}:=x_{0}$ and proved that $\left(x_{n, \alpha}^{\delta}\right)$ converges to the unique solution $x_{\alpha}^{\delta}$ of (1.3) under the following Assumptions.

Assumpion 1.1. There exists $r_{0}>0$ such that $B_{r_{0}}(\hat{x}) \subseteq D(F)$ and $F$ is Fréchet differentiable at all $x \in B_{r_{0}}(\hat{x})$.

Assumpion 1.2. There exists a constant $L>0$ such that, for every $x, u \in B_{r_{0}}(\hat{x})$ and $v \in X$, there exists an element $\Phi(x, u, v) \in X$ satisfying

$$
\left[F^{\prime}(x)-F^{\prime}(u)\right] v=F^{\prime}(u) \Phi(x, u, v), \quad\|\Phi(x, u, v)\| \leq L\|v\|,
$$

for all $x, u \in B_{r_{0}}(\hat{x})$.

Assumpion 1.3. There exists a continuous, strictly monotonically increasing function $\varphi:(0, a] \rightarrow(0, \infty)$ with $a \geq\left\|F^{\prime}(\hat{x})\right\|$ satisfying $\lim _{\lambda \rightarrow 0} \varphi(\lambda)=0$ and a vector $v \in X$ with $\|v\| \leq 1$ such that

$$
x_{0}-\hat{x}=\varphi\left(F^{\prime}(\hat{x})\right) v
$$

and

$$
\sup _{\lambda \geq 0} \frac{\alpha \varphi(\lambda)}{\lambda+\alpha} \leq \varphi(\alpha), \forall \alpha \in(0, a] .
$$

The main drawback of the method considered in [9] is that the initial guess $x_{0}$ of the iterative sequence $\left(x_{n, \alpha}^{\delta}\right)$ is highly dependent on $l_{0}$ (see Lemma 2.4 and Theorem 2.6 in [9]), so it is hard to obtain such an initial guess $x_{0}$ when $l_{0}$ is not small enough. One of the purposes of this paper is to overcome this drawback.

In this paper we use the following modified form of Assumption 1.2.

Assumpion 1.4. Let $x_{0} \in B_{r}(\hat{x})$ be fixed. There exists a constant $l_{0}>0$ such that, for every $x, x_{0} \in B_{r_{0}}(\hat{x})$ and $v \in X$, there exists an element $\Phi\left(x, x_{0}, v\right) \in X$ satisfying

$$
\left[F^{\prime}(x)-F^{\prime}\left(x_{0}\right)\right] v=F^{\prime}\left(x_{0}\right) \Phi\left(x, x_{0}, v\right), \quad\left\|\Phi\left(x, x_{0}, v\right)\right\| \leq l_{0}\|v\|\left\|x-x_{0}\right\|,
$$

for all $x \in B_{r_{0}}(\hat{x})$ and $v \in X$. 
From Assumption 1.4, one sees that the first hypotheses in Assumption 1.4 is weaker but the second hypotheses is stronger (but more practical) than the corresponding ones in Assumption 1.2. Hence Assumption 1.4 is stronger than Assumption 1.2. The autoconvolution problem discussed in [10] is an example of the nonlinear ill-posed problem satisfying Assumption 1.2 but not Assumption 1.4.

Further note that $l_{0} \leq L$ holds in general and $\frac{L}{l_{0}}$ can be arbitrarily large $[1,2,3,4,5]$. The results in [9] really require Assumption 1.4 not Assumption 1.2. If $l_{0}=L$, the results of this paper coincide with the results in [9]. Otherwise, i.e., if $l_{0}<L$, then our convergence results are better under weaker majorizing sequences. The error estimates are tighter and the information on the location of the solution as well at least as precise and the stopping rule at least as tight. Hence, the applicability of method (1.5) has been extended under less computational cost since, in practice, computing $L$ is more expensive (if at all possible) than computing $l_{0}$.

The main advantage of using the stronger Assumption 1.4 is that the majorizing sequence we are going to use in this paper is independent of the regularization parameter $\alpha$. Further the majorizing sequence gives an a priori error estimate which can be used to determine the number of iterations needed to achieve a prescribed solution accuracy before actual computation takes place.

Remark 1.1. It can be seen that functions

$$
\varphi(\lambda)=\lambda^{v}, \lambda>0
$$

for $0<v \leq 1$ and

$$
\varphi(\lambda)= \begin{cases}\left(\ln \frac{1}{\lambda}\right)^{-p}, & 0<\lambda \leq e^{-(p+1)} \\ 0, & \text { otherwise }\end{cases}
$$

for $p \geq 0$ satisfy the above assumption (see [15]).

\section{Convergence Analysis}

To prove the main results in this paper, we consider the sequence $\left(t_{n}\right), n \geq 0$ defined iteratively by $t_{0}=0, t_{1}=\eta$

$$
t_{n+1}=t_{n}+\frac{l_{0} \eta}{(1-r)}\left(t_{n}-t_{n-1}\right)
$$

where $r \in[0,1)$ as a majorizing sequence of the sequence $\left(x_{n, \alpha}^{\delta}\right)$.

The following lemma is a essential reformulation of a Lemma in [9]. For the sake of completeness, we give its proof as well.

Lemma 2.1. Assume there exist nonnegative numbers $l_{0}, \eta$ and $r \in[0,1)$ such that

$$
\frac{l_{0}}{(1-r)} \eta \leq r
$$

Then the sequence $\left(t_{n}\right)$ defined in (2.1) is increasing, bounded above by $t^{* *}:=\frac{\eta}{1-r}$, and converges to some $t^{*}$, such that $0<t^{*} \leq \frac{\eta}{1-r}$. Moreover, for $n \geq 0$,

$$
0 \leq t_{n+1}-t_{n} \leq r\left(t_{n}-t_{n-1}\right) \leq r^{n} \eta
$$

and

$$
t^{*}-t_{n} \leq \frac{r^{n}}{1-r} \eta
$$


Proof. Since the result holds for $\eta=0, l_{0}=0$ or $r=0$, we assume that $l_{0} \neq 0, \eta \neq 0$ and $r \neq 0$. Observe that $t_{1}-t_{0}=\eta \geq 0$. We assume that $t_{i+1}-t_{i} \geq 0$, for all $i \leq k$ for some $k$. Hence,

$$
t_{k+2}-t_{k+1}=\frac{l_{0} \eta}{(1-r)}\left(t_{k+1}-t_{k}\right) \geq 0
$$

and $t_{n+1}-t_{n} \geq 0$ for all $n \geq 0$. From $\frac{l_{0} \eta}{(1-r)} \leq r$, estimate (2.3) follows from (2.1). Further observe that

$$
\begin{aligned}
t_{k+1} & \leq t_{k}+r\left(t_{k}-t_{k-1}\right) \\
& \leq \cdots \\
& \leq \eta+r \eta+\cdots+r^{k} \eta \\
& <\frac{\eta}{1-r} .
\end{aligned}
$$

Hence $\left(t_{n}\right)$ is bounded above by $\frac{\eta}{1-r}$ and nondecreasing. So, it converges to some $t^{*} \leq \frac{\eta}{1-r}$, and

$$
t^{*}-t_{n}=\lim _{i \rightarrow \infty} t_{n+i}-t_{n} \leq \lim _{i \rightarrow \infty} \sum_{j=0}^{i-1}\left(t_{n+1+j}-t_{n+j}\right) \leq \frac{r^{n}}{1-r} \eta .
$$

This completes the proof of the Lemma.

To prove the convergence of the sequence $\left(x_{n, \alpha}^{\delta}\right)$ defined in (1.5), we introduce the following notations. Let $R_{\alpha}\left(x_{0}\right):=F^{\prime}\left(x_{0}\right)+\alpha I$ and

$$
G(x):=x-R_{\alpha}\left(x_{0}\right)^{-1}\left[F(x)-y^{\delta}+\alpha\left(x-x_{0}\right)\right] .
$$

Note that $G\left(x_{n, \alpha}^{\delta}\right)=x_{n+1, \alpha}^{\delta}$ and

$$
\left\|R_{\alpha}\left(x_{0}\right)^{-1} F^{\prime}\left(x_{0}\right)\right\| \leq 1 .
$$

The following Lemma based on the Assumption 1.4 will be used later.

Lemma 2.2. For $u, v, x_{0} \in B_{r_{0}}(\hat{x})$,

$$
F(v)-F(u)-F^{\prime}\left(x_{0}\right)(v-u)=F^{\prime}\left(x_{0}\right) \int_{0}^{1} \Phi\left(u+t(v-u), x_{0}, v-u\right) d t .
$$

Proof. Using the Fundamental Theorem of Integration, for $u, v, x_{0} \in B_{r_{0}}(\hat{x})$ we have

$$
F(v)-F(u)=\int_{0}^{1} F^{\prime}(u+t(v-u))(v-u) d t .
$$

From Assumption 1.4, we have

$$
F(v)-F(u)-F^{\prime}\left(x_{0}\right)(v-u)=F^{\prime}\left(x_{0}\right) \int_{0}^{1} \Phi\left(u+t(v-u), x_{0}, v-u\right) d t .
$$

This completes the proof of the Lemma.

Hereafter we assume that $\left\|x_{0}-\hat{x}\right\| \leq \rho$ and

$$
\frac{l_{0}}{2} \rho^{2}+\rho+\frac{\delta}{\alpha} \leq \eta \leq \min \left\{\frac{r(1-r)}{l_{0}}, r_{0}(1-r)\right\} .
$$


Theorem 2.1. Suppose that (2.1) holds. Let the assumptions in Lemma 2.1 with $\eta$ be as in (2.7) and Assumption 1.4 be satisfied. Then the sequence $\left(x_{n, \alpha}^{\delta}\right)$ defined in (1.5) is well defined and $x_{n, \alpha}^{\delta} \in B_{t^{*}}\left(x_{0}\right)$ for all $n \geq 0$. Further $\left(x_{n, \alpha}^{\delta}\right)$ is a Cauchy sequence in $B_{t^{*}}\left(x_{0}\right)$ (converges to $x_{\alpha}^{\delta} \in \overline{B_{t^{*}}\left(x_{0}\right)} \subset B_{t^{* *}}\left(x_{0}\right)$ ) and $F\left(x_{\alpha}^{\delta}\right)+\alpha\left(x_{\alpha}^{\delta}-x_{0}\right)=y^{\delta}$. Moreover, the following estimate hold, for all $n \geq 0$,

$$
\left\|x_{n+1, \alpha}^{\delta}-x_{n, \alpha}^{\delta}\right\| \leq t_{n+1}-t_{n}
$$

and

$$
\left\|x_{n, \alpha}^{\delta}-x_{\alpha}^{\delta}\right\| \leq t^{*}-t_{n} \leq \frac{r^{n} \eta}{(1-r)}
$$

Proof. Let $G$ be as in (2.5). Then, for $u, v \in B_{t^{*}}\left(x_{0}\right)$,

$$
\begin{aligned}
G(u)-G(v)= & u-v-R_{\alpha}\left(x_{0}\right)^{-1}\left[F(u)-y^{\delta}+\alpha\left(u-x_{0}\right)\right] \\
& +R_{\alpha}\left(x_{0}\right)^{-1}\left[F(v)-y^{\delta}+\alpha\left(v-x_{0}\right)\right] \\
= & R_{\alpha}\left(x_{0}\right)^{-1}\left[R_{\alpha}\left(x_{0}\right)(u-v)-(F(u)-F(v))\right] \\
& +\alpha R_{\alpha}\left(x_{0}\right)^{-1}(v-u) \\
= & R_{\alpha}\left(x_{0}\right)^{-1}\left[F^{\prime}\left(x_{0}\right)(u-v)-(F(u)-F(v))+\alpha(u-v)\right] \\
& +\alpha R_{\alpha}\left(x_{0}\right)^{-1}(v-u) \\
= & R_{\alpha}\left(x_{0}\right)^{-1}\left[F^{\prime}\left(x_{0}\right)(u-v)-(F(u)-F(v))\right] .
\end{aligned}
$$

Using Lemma 2.2, Assumption 1.4, (2.6) and (2.7) we have

$$
\|G(u)-G(v)\| \leq l_{0} t^{*}\|u-v\| .
$$

Now we prove that the sequence $\left(t_{n}\right)$ defined in Lemma 2.1 is a majorizing sequence of $\left(x_{n, \alpha}^{\delta}\right)$ and $x_{n, \alpha}^{\delta} \in B_{t^{*}}\left(x_{0}\right)$, for all $n \geq 0$. Since $F(\hat{x})=y$, one has

$$
\begin{aligned}
\left\|x_{1, \alpha}^{\delta}-x_{0}\right\|= & \left\|R_{\alpha}\left(x_{0}\right)^{-1}\left(F\left(x_{0}\right)-y^{\delta}\right)\right\| \\
= & \left\|R_{\alpha}\left(x_{0}\right)^{-1}\left(F\left(x_{0}\right)-y+y-y^{\delta}\right)\right\| \\
= & \| R_{\alpha}\left(x_{0}\right)^{-1}\left(F\left(x_{0}\right)-F(\hat{x})-F^{\prime}\left(x_{0}\right)\left(x_{0}-\hat{x}\right)\right. \\
& \left.+F^{\prime}\left(x_{0}\right)\left(x_{0}-\hat{x}\right)+y-y^{\delta}\right) \| \\
\leq & \left\|R_{\alpha}\left(x_{0}\right)^{-1}\left(F\left(x_{0}\right)-F(\hat{x})-F^{\prime}\left(x_{0}\right)\left(x_{0}-\hat{x}\right)\right)\right\| \\
& +\left\|R_{\alpha}\left(x_{0}\right)^{-1} F^{\prime}\left(x_{0}\right)\left(x_{0}-\hat{x}\right)\right\|+\left\|R_{\alpha}\left(x_{0}\right)^{-1}\left(y-y^{\delta}\right)\right\| \\
\leq & \left\|R_{\alpha}\left(x_{0}\right)^{-1} F^{\prime}\left(x_{0}\right) \int_{0}^{1} \Phi\left(\hat{x}+t\left(x_{0}-\hat{x}\right), x_{0},\left(x_{0}-\hat{x}\right)\right) d t\right\| \\
& \left.+\| R_{\alpha}\left(x_{0}\right)^{-1} F^{\prime}\left(x_{0}\right)\right)\left(x_{0}-\hat{x}\right) \|+\frac{\delta}{\alpha} \\
\leq & \frac{l_{0}}{2}\left\|x_{0}-\hat{x}\right\|^{2}+\left\|x_{0}-\hat{x}\right\|+\frac{\delta}{\alpha} \\
\leq & \frac{l_{0}}{2} \rho^{2}+\rho+\frac{\delta}{\alpha} \leq \eta=t_{1}-t_{0} .
\end{aligned}
$$

The last but one step follows from Assumption 1.4. Assume that

$$
\left\|x_{i+1, \alpha}^{\delta}-x_{i, \alpha}^{\delta}\right\| \leq t_{i+1}-t_{i}, \quad \forall i \leq k
$$


for some $k$. Then

$$
\begin{aligned}
\left\|x_{k+1, \alpha}^{\delta}-x_{0}\right\| & =\left\|x_{k+1, \alpha}^{\delta}-x_{k, \alpha}^{\delta}+x_{k, \alpha}^{\delta}-x_{k-1, \alpha}^{\delta}+\cdots+x_{1, \alpha}^{\delta}-x_{0}\right\| \\
& \leq\left\|x_{k+1, \alpha}^{\delta}-x_{k, \alpha}^{\delta}\right\|+\left\|x_{k, \alpha}^{\delta}-x_{k-1, \alpha}^{\delta}\right\|+\cdots+\left\|x_{1, \alpha}^{\delta}-x_{0}\right\| \\
& \leq t_{k+1}-t_{k}+t_{k}-t_{k-1}+\cdots+t_{1}-t_{0} \\
& =t_{k+1} \leq t^{*} .
\end{aligned}
$$

So $x_{i+1, \alpha}^{\delta} \in B_{t^{*}}\left(x_{0}\right)$ for all $i \leq k$. Hence, by (2.10) and (2.11), one has

$$
\left\|x_{k+2, \alpha}^{\delta}-x_{k+1, \alpha}^{\delta}\right\| \leq l_{0} t^{*}\left\|x_{k+1, \alpha}^{\delta}-x_{k, \alpha}^{\delta}\right\| \leq \frac{l_{0} \eta}{(1-r)}\left(t_{k+1}-t_{k}\right)=t_{k+2}-t_{k+1} .
$$

Thus by induction, $\left\|x_{n+1, \alpha}^{\delta}-x_{n, \alpha}^{\delta}\right\| \leq t_{n+1}-t_{n}$ for all $n \geq 0$. Hence $\left(t_{n}\right), n \geq 0$ is a majorizing sequence of $\left(x_{n, \alpha}^{\delta}\right)$. In particular, $\left\|x_{n, \alpha}^{\delta}-x_{0}\right\| \leq t_{n} \leq t^{*}$, i.e., $x_{n, \alpha}^{\delta} \in B_{t^{*}}\left(x_{0}\right)$, for all $n \geq 0$. Hence, $\left(x_{n, \alpha}^{\delta}\right)$ is a Cauchy sequence and converges to some $x_{\alpha}^{\delta} \in \overline{B_{t^{*}}\left(x_{0}\right)} \subset B_{t^{* *}}\left(x_{0}\right)$ and

$$
\left\|x_{\alpha}^{\delta}-x_{n, \alpha}^{\delta}\right\| \leq t^{*}-t_{n} \leq \frac{r^{n} \eta}{(1-r)}
$$

Letting $n \rightarrow \infty$ in (1.5), we obtain $F\left(x_{\alpha}^{\delta}\right)+\alpha\left(x_{\alpha}^{\delta}-x_{0}\right)=y^{\delta}$. This completes the proof of the Theorem.

\section{ERror Bounds Under SOURCE CONDITIONS}

We will use the error estimates in the following Proposition, which can be found in [20] for our error analysis.

Proposition 3.1. [20, Proposition 3.1] Let $\hat{x} \in D(F)$ be a solution of (1.1) and let $F: D(F) \subseteq X \mapsto X$ be a monotone operator in $X$. Let $x_{\alpha}$ be the unique solution of

$$
F(x)+\alpha\left(x-x_{0}\right)=y
$$

and let $x_{\alpha}^{\delta}$ be the unique solution of (1.3). Then

$$
\left\|x_{\alpha}^{\delta}-x_{\alpha}\right\| \leq \frac{\delta}{\alpha}
$$

and

$$
\left\|x_{\alpha}-\hat{x}\right\| \leq\left\|x_{0}-\hat{x}\right\|
$$

To obtain an error estimate for $\left\|x_{\alpha}^{\delta}-\hat{x}\right\|$, it is enough to obtain an error estimate for $\left\|x_{\alpha}^{\delta}-x_{\alpha}\right\|$ and $\left\|x_{\alpha}-\hat{x}\right\|$.

Let us introduce the following operators:

$$
A:=F^{\prime}(\hat{x})
$$

and

$$
M_{\alpha}:=\int_{0}^{1} F^{\prime}\left(\hat{x}+t\left(x_{\alpha}-\hat{x}\right)\right) d t .
$$

Using the Mean Value Theorem in Integral form, we have

$$
F\left(x_{\alpha}\right)-F(\hat{x})=M_{\alpha}\left(x_{\alpha}-\hat{x}\right) .
$$

The following Theorem gives an estimate for $\left\|x_{\alpha}-\hat{x}\right\|$. 
Theorem 3.1. Let $x_{\alpha}$ be the unique solution of (3.1) and let the Assumptions 1.1, 1.3 and 1.4 be satisfied. Then

$$
\left\|x_{\alpha}-\hat{x}\right\| \leq\left(1+l_{0} r_{0}\right) c_{\varphi} \varphi(\alpha)
$$

Proof. Since $F\left(x_{\alpha}\right)+\alpha\left(x_{\alpha}-x_{0}\right)=y$, for any $\alpha>0$, we obtain from (3.5) that

$$
M_{\alpha}\left(x_{\alpha}-\hat{x}\right)+\alpha\left(x_{\alpha}-\hat{x}\right)=\alpha\left(x_{0}-\hat{x}\right) .
$$

Hence

$$
\begin{aligned}
x_{\alpha}-\hat{x}= & \left(M_{\alpha}+\alpha I\right)^{-1} \alpha\left(x_{0}-\hat{x}\right) \\
= & {\left[\left(M_{\alpha}+\alpha I\right)^{-1}-(A+\alpha I)^{-1}\right] \alpha\left(x_{0}-\hat{x}\right)+\alpha(A+\alpha I)^{-1}\left(x_{0}-\hat{x}\right) } \\
= & \left(M_{\alpha}+\alpha I\right)^{-1}\left(A-M_{\alpha}\right) \alpha(A+\alpha I)^{-1}\left(x_{0}-\hat{x}\right)+\alpha(A+\alpha I)^{-1}\left(x_{0}-\hat{x}\right) \\
= & \left(M_{\alpha}+\alpha I\right)^{-1} M_{\alpha} \Phi\left(\hat{x}, \hat{x}+t\left(x_{\alpha}-\hat{x}\right), \alpha(A+\alpha I)^{-1}\left(x_{0}-\hat{x}\right)\right) \\
& +\alpha(A+\alpha I)^{-1}\left(x_{0}-\hat{x}\right)
\end{aligned}
$$

which follows from Assumption 1.4. From Proposition 3.1, Assumptions 1.1, 1.3 and 1.4 we have

$$
\begin{aligned}
\left\|x_{\alpha}-\hat{x}\right\|= & \|\left(M_{\alpha}+\alpha I\right)^{-1} M_{\alpha} \Phi\left(\hat{x}, \hat{x}+t\left(x_{\alpha}-\hat{x}\right), \alpha(A+\alpha I)^{-1}\left(x_{0}-\hat{x}\right)\right) \\
& +\alpha(A+\alpha I)^{-1}\left(x_{0}-\hat{x}\right) \| \\
\leq & \left\|\left(M_{\alpha}+\alpha I\right)^{-1} M_{\alpha} \Phi\left(\hat{x}, \hat{x}+t\left(x_{\alpha}-\hat{x}\right), \alpha(A+\alpha I)^{-1}\left(x_{0}-\hat{x}\right)\right)\right\| \\
& +\left\|\alpha(A+\alpha I)^{-1}\left(x_{0}-\hat{x}\right)\right\| \\
\leq & \left(l_{0} r_{0}+1\right) c_{\varphi} \varphi(\alpha) .
\end{aligned}
$$

This completes the proof.

Combining the estimates in Theorem 2.1, (3.2) and (3.6), we obtain the following Theorem.

Theorem 3.2. Let $x_{n, \alpha}^{\delta}$ be as in (1.5) and let the assumptions in Theorem 2.1, (3.2) and (3.6) be satisfied. Then

$$
\left\|x_{n, \alpha}^{\delta}-\hat{x}\right\| \leq \frac{r^{n} \eta}{1-r}+\frac{\delta}{\alpha}+\left(l_{0} r_{0}+1\right) c_{\varphi} \varphi(\alpha)
$$

Let

$$
n_{\delta}:=\min \left\{n: r^{n} \leq \delta\right\}
$$

and

$$
C:=\max \left\{\frac{\eta}{1-r}+1,\left(l_{0} r_{0}+1\right) c_{\varphi}\right\}
$$

Theorem 3.3. Let $x_{n, \alpha}^{\delta}$ be as in (1.5) and let the assumptions in Theorem 3.2 be satisfied. Let $n_{\delta}$ be as in (3.8) and let $C$ be as in (3.9). Then, for all $0<\alpha \leq 1$,

$$
\left\|x_{n_{\delta}, \alpha}^{\delta}-\hat{x}\right\| \leq C\left(\varphi(\alpha)+\frac{\delta}{\alpha}\right) .
$$


3.1. A priori choice of the parameter. Note that the error $\varphi(\alpha)+\frac{\delta}{\alpha}$ in (3.10) is of optimal order if $\alpha_{\delta}:=\alpha(\delta)$ satisfies $\alpha_{\delta} \varphi\left(\alpha_{\delta}\right)=\delta$. Using $\psi(\lambda):=\lambda \varphi^{-1}(\lambda), 0<\lambda \leq a$, we have

$$
\delta=\alpha_{\delta} \varphi\left(\alpha_{\delta}\right)=\psi\left(\varphi\left(\alpha_{\delta}\right)\right) .
$$

Hence, $\alpha_{\delta}=\varphi^{-1}\left(\psi^{-1}(\delta)\right)$. Using (3.10), we have the following.

Theorem 3.4. Let $\psi(\lambda):=\lambda \varphi^{-1}(\lambda)$ for $0<\lambda \leq a$, and let the assumptions in Theorem 3.3 holds. For $\delta>0$, let $\alpha:=\alpha_{\delta}=\varphi^{-1}\left(\psi^{-1}(\delta)\right)$. Let $n_{\delta}$ be as in (3.8). Then

$$
\left\|x_{n_{\delta}, \alpha}^{\delta}-\hat{x}\right\|=\bigcirc\left(\psi^{-1}(\delta)\right) .
$$

3.2. An adaptive choice of the parameter. In this subsection, we present a parameter choice rule based on the adaptive method studied in $[14,16]$.

In practice, the regularization parameter $\alpha$ is often selected from some finite set

$$
D_{M}(\alpha):=\left\{\alpha_{i}=\mu^{i} \alpha_{0}, i=0,1, \cdots, M\right\},
$$

where $\mu>1$ and $M$ is such that $\alpha_{M}<1 \leq \alpha_{M+1}$. We choose $\alpha_{0}:=\sqrt{\delta}$ because in general $\varphi(\lambda)=$ $\lambda^{v}, 0<v \leq 1$. In this case, the best possible error estimate is of order $\bigcirc(\sqrt{\delta})$. From Theorem 3.4, it follows that such an accuracy cannot be guaranteed for $\alpha<\sqrt{\delta}$. Let

$$
n_{M}:=\min \left\{n: r^{n} \leq \delta\right\} .
$$

Then, for $i=0,1, \cdots, M$,

$$
\left\|x_{n_{M}, \alpha_{i}}^{\delta}-x_{\alpha_{i}}^{\delta}\right\| \leq \frac{\delta}{\alpha_{i}}, \quad \forall i=0,1, \cdots M
$$

Let $x_{i}:=x_{n_{M}, \alpha_{i}}^{\delta}$. We selects $\alpha=\alpha_{i}$ from $D_{M}(\alpha)$ and operates only with corresponding $x_{i}, i=0,1, \cdots, M$.

Theorem 3.5. Assume that there exists $i \in\{0,1,2, \cdots, M\}$ such that $\varphi\left(\alpha_{i}\right) \leq \frac{\delta}{\alpha_{i}}$. Let assumptions of Theorem 3.3 and Theorem 3.4 hold and let

$$
\begin{gathered}
l:=\max \left\{i: \varphi\left(\alpha_{i}\right) \leq \frac{\delta}{\alpha_{i}}\right\}<M, \\
k:=\max \left\{i:\left\|x_{i}-x_{j}\right\| \leq 4 C \frac{\delta}{\alpha_{j}}, j=0,1,2, \cdots . i\right\} .
\end{gathered}
$$

Then, $l \leq k$, $\left\|\hat{x}-x_{k}\right\| \leq c \psi^{-1}(\delta)$, where $c=6 C \mu$.

Proof. To see that $l \leq k$, it is enough to show that, for each $i \in\{1,2, \cdots, M\}$,

$$
\varphi\left(\alpha_{i}\right) \leq \frac{\delta}{\alpha_{i}} \Longrightarrow\left\|x_{i}-x_{j}\right\| \leq 4 C \frac{\delta}{\alpha_{j}}, \quad \forall j=0,1, \cdots, i .
$$

For $j \leq i$, we conclude from (3.10)that

$$
\begin{aligned}
\left\|x_{i}-x_{j}\right\| & \leq\left\|x_{i}-\hat{x}\right\|+\left\|\hat{x}-x_{j}\right\| \\
& \leq C\left(\varphi\left(\alpha_{i}\right)+\frac{\delta}{\alpha_{i}}\right)+C\left(\varphi\left(\alpha_{j}\right)+\frac{\delta}{\alpha_{j}}\right) \\
& \leq 2 C \frac{\delta}{\alpha_{i}}+2 C \frac{\delta}{\alpha_{j}} . \\
& \leq 4 C \frac{\delta}{\alpha_{j}} .
\end{aligned}
$$


Thus the relation $l \leq k$ is proved. Next we observe that

$$
\begin{aligned}
\left\|\hat{x}-x_{k}\right\| & \leq\left\|\hat{x}-x_{l}\right\|+\left\|x_{l}-x_{k}\right\| \\
& \leq C\left(\varphi\left(\alpha_{l}\right)+\frac{\delta}{\alpha_{l}}\right)+4 C \frac{\delta}{\alpha_{l}} \\
& \leq 6 C \frac{\delta}{\alpha_{l}} .
\end{aligned}
$$

Since $\alpha_{\delta} \leq \alpha_{l+1} \leq \mu \alpha_{l}$, one has

$$
\frac{\delta}{\alpha_{l}} \leq \mu \frac{\delta}{\alpha_{\delta}}=\mu \varphi\left(\alpha_{\delta}\right)=\mu \psi^{-1}(\delta)
$$

This completes the proof.

\section{IMPLEMENTATION OF THE ADAPTIVE CHOICE RULE}

In this section, we provide an algorithm for the determination of a parameter fulfilling the balancing principle (3.14) and provide a starting point for the iteration (1.5) to approximate the unique solution $x_{\alpha}^{\delta}$ of (1.3). The choice of the starting point involves the following steps:

- Choose $\alpha_{0}=\sqrt{\delta}$ and $\mu>1$.

- Choose $x_{0} \in D(F)$ such that $\left\|x_{0}-\hat{x}\right\| \leq \rho$.

- Choose $\eta$ satisfying (2.7).

The choice of the stopping index $n_{M}$ involves the following step:

- Choose $n_{M}$ such that $n_{M}=\min \left\{n: r^{n} \leq \delta\right\}$.

Finally, the adaptive algorithm associated with the choice of the parameter specified in Theorem 3.5 involves the following steps.

\subsection{The algorithm.}

- Set $i \leftarrow 0$.

- Solve $x_{i}:=x_{n_{M}, \alpha_{i}}^{\delta}$ via iteration (1.5).

- If $\left\|x_{i}-x_{j}\right\|>4 C \frac{\sqrt{\delta}}{\mu^{j}}, \quad j \leq i$, then we take $k=i-1$.

- Set $i=i+1$ and return to step 2 .

\section{The Iteratively Regularized Projection Method}

Let $H$ be a bounded subset of positive real numbers such that zero is a limit point of $H$. Let $\left\{P_{h}\right\}_{h \in H}$ be a family of orthogonal projections from $X$ into itself. Let

$$
\Gamma_{h}:=\left\|\left(I-P_{h}\right) F^{\prime}\left(x_{0}\right)\right\|
$$

and

$$
\gamma_{h}:=\left\|F^{\prime}\left(P_{h} x_{0}\right)\left(I-P_{h}\right)\right\|
$$

We assume that

$$
b_{h}:=\left\|\left(I-P_{h}\right) x_{0}\right\| \rightarrow 0, \quad \text { as } \rightarrow 0 .
$$

The above assumption is satisfied if $P_{h} \rightarrow I$ pointwise. Let $\left(t_{n, h}\right), n \geq 0$ be defined iteratively by $t_{0, h}=$ $0, t_{1, h}=\eta_{h}$

$$
t_{n+1, h}=t_{n, h}+\left(1+\frac{\gamma_{h}}{\alpha}\right) \frac{l_{0} \eta_{h}}{\left(1-r_{h}\right)}\left(t_{n, h}-t_{n-1, h}\right)
$$


where $l_{0}, \alpha$ and $r_{h} \in[0,1)$ are nonnegative numbers with $\left(1+\frac{\gamma_{h}}{\alpha}\right) \frac{l_{0}}{\left(1-r_{h}\right)} \eta_{h} \leq r_{h}$. We need the following Lemma. Its proof is analogous to the proof of Lemma 2.1. So, we omit the proof.

Lemma 5.1. Assume there exist nonnegative numbers $l_{0}, \alpha$ and $r_{h} \in[0,1)$ such that

$$
\left(1+\frac{\gamma_{h}}{\alpha}\right) \frac{l_{0}}{\left(1-r_{h}\right)} \eta_{h} \leq r_{h}
$$

Then the sequence $\left(t_{n, h}\right)$ defined in (5.4) is increasing, bounded above by $t_{h}^{* *}:=\frac{\eta_{h}}{1-r_{h}}$, and converges to some $t_{h}^{*}$, such that $0<\frac{\eta_{h}}{1-r_{h}}$. Moreover, for $n \geq 0$,

$$
0 \leq t_{n+1, h}-t_{n, h} \leq r_{h}\left(t_{n, h}-t_{n-1, h}\right) \leq r_{h}^{n} \eta_{h}
$$

and

$$
t_{h}^{*}-t_{n, h} \leq \frac{r_{h}^{n}}{1-r_{h}} \eta_{h}
$$

We considered the following iteratively regularized projection method

$$
x_{n+1, \alpha}^{h, \delta}:=x_{n, \alpha}^{h, \delta}-\left(P_{h} F^{\prime}\left(P_{h} x_{0}\right)+\alpha I\right)^{-1} P_{h}\left(F\left(x_{n, \alpha}^{h, \delta}\right)-y^{\delta}+\alpha\left(x_{n, \alpha}^{h, \delta}-x_{0}\right)\right),
$$

where $x_{0, \alpha}^{h, \delta}:=P_{h} x_{0}$ for $\left(x_{n, \alpha}^{h, \delta}\right)$ in a finite dimensional subspace $X_{h}$ of $X$.

Next we prove that sequence $\left(t_{n, h}\right)$ is a majorizing sequence of $\left(x_{n, \alpha}^{h, \delta}\right)$. Let

$$
\left(1+\frac{\gamma_{h}}{\alpha}\right)\left(\frac{l_{0}}{2}\left(b_{h}+\rho\right)^{2}+b_{h}+\rho\right)+\frac{\delta}{\alpha} \leq \eta_{h} \leq \min \left\{\frac{r_{h}\left(1-r_{h}\right)}{l_{0}\left(1+\gamma_{h} / \alpha\right)}, r_{0}\left(1-r_{h}\right)\right\} .
$$

Theorem 5.1. Let the assumptions in Lemma 5.1 with $\eta_{h}$ be as in (5.9) and let Assumption 1.4 be satisfied. Then the sequence $\left(t_{n, h}\right)$ defined in (5.4) is a majorizing sequence of the sequence $\left(x_{n, \alpha}^{h, \delta}\right)$ defined in (5.8) and $x_{n, \alpha}^{h, \delta} \in B_{t_{h}^{*}}\left(P_{h} x_{0}\right)$ for all $n \geq 0$.

Proof. Let

$$
G(x)=x-R_{\alpha}\left(P_{h} x_{0}\right)^{-1}\left[F(x)-y^{\delta}+\alpha\left(x-x_{0}\right)\right]
$$

where $R_{\alpha}\left(P_{h} x_{0}\right)^{-1}=\left(P_{h} F^{\prime}\left(P_{h} x_{0}\right) P_{h}+\alpha P_{h}\right)^{-1}$. Since

$$
R_{\alpha}\left(P_{h} x_{0}\right)^{-1}=R_{\alpha}\left(P_{h} x_{0}\right)^{-1} P_{h}=P_{h} R_{\alpha}\left(P_{h} x_{0}\right)^{-1},
$$

for $u, v \in B_{t_{h}^{*}}\left(P_{h} x_{0}\right)$, one has

$$
\begin{aligned}
G(u)-G(v) & =u-v-R_{\alpha}\left(P_{h} x_{0}\right)^{-1}\left[F(u)-y^{\delta}+\alpha\left(u-x_{0}\right)\right]+R_{\alpha}\left(P_{h} x_{0}\right)^{-1}\left[F(v)-y^{\delta}+\alpha\left(v-x_{0}\right)\right] \\
& =R_{\alpha}\left(P_{h} x_{0}\right)^{-1}\left[R_{\alpha}\left(P_{h} x_{0}\right)(u-v)-(F(u)-F(v))\right]+\alpha R_{\alpha}\left(P_{h} x_{0}\right)^{-1}(v-u) \\
& =R_{\alpha}\left(P_{h} x_{0}\right)^{-1}\left[F^{\prime}\left(P_{h} x_{0}\right) P_{h}(u-v)-(F(u)-F(v))+\alpha(u-v)\right]+\alpha R_{\alpha}\left(P_{h} x_{0}\right)^{-1}(v-u) \\
& =R_{\alpha}\left(P_{h} x_{0}\right)^{-1}\left[F^{\prime}\left(P_{h} x_{0}\right) P_{h}(u-v)-(F(u)-F(v))\right] .
\end{aligned}
$$


Since $G\left(x_{n, \alpha}^{h, \delta}\right)=x_{n+1, \alpha}^{h, \delta}$ and $P_{h}\left(x_{n, \alpha}^{h, \delta}-x_{n-1, \alpha}^{h, \delta}\right)=\left(x_{n, \alpha}^{h, \delta}-x_{n-1, \alpha}^{h, \delta}\right)$, we have from Lemma 2.2 that

$$
\begin{aligned}
\left(x_{n+1, \alpha}^{h, \delta}-x_{n, \alpha}^{h, \delta}\right) & =G\left(x_{n, \alpha}^{h, \delta}\right)-G\left(x_{n-1, \alpha}^{h, \delta}\right) \\
& =R_{\alpha}\left(P_{h} x_{0}\right)^{-1}\left[F^{\prime}\left(P_{h} x_{0}\right)\left(x_{n, \alpha}^{h, \delta}-x_{n-1, \alpha}^{h, \delta}\right)-\left(F\left(x_{n, \alpha}^{h, \delta}\right)-F\left(x_{n-1, \alpha}^{h, \delta}\right)\right)\right] \\
& =R_{\alpha}\left(P_{h} x_{0}\right)^{-1} F^{\prime}\left(P_{h} x_{0}\right) \int_{0}^{1} \Phi\left(x_{n, \alpha}^{h, \delta}+t\left(x_{n-1, \alpha}^{h, \delta}-x_{n, \alpha}^{h, \delta}\right), P_{h} x_{0}, x_{n-1, \alpha}^{h, \delta}-x_{n, \alpha}^{h, \delta}\right) d t \\
& =R_{\alpha}\left(P_{h} x_{0}\right)^{-1}\left[F^{\prime}\left(P_{h} x_{0}\right) P_{h}+F^{\prime}\left(P_{h} x_{0}\right)\left(I-P_{h}\right)\right] \\
& \times \int_{0}^{1} \Phi\left(x_{n, \alpha}^{h, \delta}+t\left(x_{n-1, \alpha}^{h, \delta}-x_{n, \alpha}^{h, \delta}\right), P_{h} x_{0}, x_{n-1, \alpha}^{h, \delta}-x_{n, \alpha}^{h, \delta}\right) d t .
\end{aligned}
$$

Using Assumption 1.4 and

$$
\left\|R_{\alpha}\left(P_{h} x_{0}\right)^{-1}\left[F^{\prime}\left(P_{h} x_{0}\right) P_{h}+F^{\prime}\left(P_{h} x_{0}\right)\left(I-P_{h}\right)\right]\right\| \leq 1+\frac{\gamma_{h}}{\alpha},
$$

we have

$$
\left\|x_{n+1, \alpha}^{h, \delta}-x_{n, \alpha}^{h, \delta}\right\| \leq\left(1+\frac{\gamma_{h}}{\alpha}\right) l_{0}\left\|x_{n, \alpha}^{h, \delta}+t\left(x_{n-1, \alpha}^{h, \delta}-x_{n, \alpha}^{h, \delta}\right)-P_{h} x_{0}\right\|\left\|x_{n, \alpha}^{h, \delta}-x_{n-1, \alpha}^{h, \delta}\right\| .
$$

Now we prove that the sequence $\left(t_{n, h}\right)$ defined in (5.4) is a majorizing sequence of $\left(x_{n, \alpha}^{h, \delta}\right)$ and $x_{n, \alpha}^{h, \delta} \in$ $B_{t_{h}^{*}}\left(P_{h} x_{0}\right)$, for all $n \geq 0$. In view of $F(\hat{x})=y$, Assumption 1.4, (5.10) and inequality $\left\|P_{h} x_{0}-\hat{x}\right\| \leq b_{h}+\rho$, one has

$$
\begin{aligned}
\left\|x_{1, \alpha}^{h, \delta}-P_{h} x_{0}\right\|= & \left\|\left(P_{h} F^{\prime}\left(P_{h} x_{0}\right)+\alpha I\right)^{-1} P_{h}\left(F\left(P_{h} x_{0}\right)-y^{\delta}\right)\right\| \\
= & \left\|\left(P_{h} F^{\prime}\left(P_{h} x_{0}\right)+\alpha I\right)^{-1} P_{h}\left(F\left(P_{h} x_{0}\right)-F(\hat{x})+y-y^{\delta}\right)\right\| \\
\leq & \left\|\left(P_{h} F^{\prime}\left(P_{h} x_{0}\right)+\alpha I\right)^{-1} P_{h}\left(F\left(P_{h} x_{0}\right)-F(\hat{x})-F^{\prime}\left(P_{h} x_{0}\right)\left(P_{h} x_{0}-\hat{x}\right)\right)\right\| \\
& +\left\|\left(P_{h} F^{\prime}\left(P_{h} x_{0}\right)+\alpha I\right)^{-1} P_{h} F^{\prime}\left(P_{h} x_{0}\right)\left(P_{h} x_{0}-\hat{x}\right)\right\| \\
& +\left\|\left(P_{h} F^{\prime}\left(P_{h} x_{0}\right)+\alpha I\right)^{-1} P_{h}\left(y-y^{\delta}\right)\right\| \\
\leq & \left(1+\frac{\gamma_{h}}{\alpha}\right)\left(\frac{l_{0}}{2}\left\|P_{h} x_{0}-\hat{x}\right\|^{2}+\left\|P_{h} x_{0}-\hat{x}\right\|\right)+\frac{\delta}{\alpha} \\
\leq & \left(1+\frac{\gamma_{h}}{\alpha}\right)\left(\frac{l_{0}}{2}\left(b_{h}+\rho\right)^{2}+b_{h}+\rho\right)+\frac{\delta}{\alpha} \\
\leq & \eta_{h} .
\end{aligned}
$$

So, $\left\|x_{1, \alpha}^{h, \delta}-P_{h} x_{0}\right\| \leq t_{1, h}-t_{0, h}$. Assume that

$$
\left\|x_{i+1, \alpha}^{h, \delta}-x_{i, \alpha}^{h, \delta}\right\| \leq t_{i+1, h}-t_{i, h}, \quad \forall i \leq k
$$

for some $k$. Then

$$
\begin{aligned}
\left\|x_{k+1, \alpha}^{h, \delta}-P_{h} x_{0}\right\| & =\left\|x_{k+1, \alpha}^{h, \delta}-x_{k, \alpha}^{h, \delta}+x_{k, \alpha}^{h, \delta}-x_{k-1, \alpha}^{h, \delta}+\cdots+x_{1, \alpha}^{h, \delta}-P_{h} x_{0}\right\| \\
& \leq\left\|x_{k+1, \alpha}^{h, \delta}-x_{k, \alpha}^{h, \delta}\right\|+\left\|x_{k, \alpha}^{h, \delta}-x_{k-1, \alpha}^{h, \delta}\right\|+\cdots+\left\|x_{1, \alpha}^{h, \delta}-P_{h} x_{0}\right\| \\
& \leq t_{k+1, h}-t_{k, h}+t_{k, h}-t_{k-1, h}+\cdots+t_{1, h}-t_{0, h} \\
& =t_{k+1, h} \leq t_{h}^{*} .
\end{aligned}
$$

So $x_{i+1, \alpha}^{h, \delta} \in B_{t_{h}^{*}}\left(P_{h} x_{0}\right)$ for all $i \leq k$. Hence,

$$
x_{k+1, \alpha}^{h, \delta}+t\left(x_{k, \alpha}^{h, \delta}-x_{k+1, \alpha}^{h, \delta}\right) \in B_{t_{h}^{*}}\left(P_{h} x_{0}\right) .
$$


By (5.11) and (5.11), we have

$$
\begin{aligned}
\left\|x_{k+2, \alpha}^{h, \delta}-x_{k+1, \alpha}^{h, \delta}\right\| & \leq l_{0}\left(1+\frac{\gamma_{h}}{\alpha}\right) t_{h}^{*}\left\|x_{k+1, \alpha}^{h, \delta}-x_{k, \alpha}^{h, \delta}\right\| \\
& =t_{k+2, h}-t_{k+1, h} .
\end{aligned}
$$

Thus, $\left\|x_{n+1, \alpha}^{h, \delta}-x_{n, \alpha}^{h, \delta}\right\| \leq t_{n+1, h}-t_{n, h}, \forall n \geq 0$. Hence $\left(t_{n, h}\right), n \geq 0$ is a majorizing sequence of $\left(x_{n, \alpha}^{h, \delta}\right)$. In particular $\left\|x_{n, \alpha}^{h, \delta}-P_{h} x_{0}\right\| \leq t_{n, h} \leq t_{h}^{*}$, i.e., $x_{n, \alpha}^{h, \delta} \in B_{t_{h}^{*}}\left(P_{h} x_{0}\right)$, for all $n \geq 0$. Hence

$$
\left\|x_{n, \alpha}^{h, \delta}-P_{h} x_{0}\right\| \leq t_{h}^{*} \leq \frac{\eta_{h}}{1-r_{h}} .
$$

This completes the proof.

Let

$$
\tilde{\tilde{r}}:=\max \left\{r, r_{h}\right\}
$$

and

$$
q:=\frac{1}{2}\left[2 \tilde{\tilde{r}}+l_{0} b_{h}\right]
$$

For $0<b_{h}<\frac{2(1-\tilde{r})}{l_{0}}, q<1$, one has the following.

Theorem 5.2. Let $x_{n, \alpha}^{h, \delta}$ be as in (5.8) and let $x_{n, \alpha}^{\delta}$ be as in (1.5). Let assumptions in Theorem 2.1 and Theorem 5.1 hold. Then

$$
\left\|x_{n, \alpha}^{h, \delta}-x_{n, \alpha}^{\delta}\right\| \leq q^{n} b_{h}+\frac{\Gamma_{h}+l_{0}\left\|F^{\prime}\left(x_{0}\right)\right\| b_{h}}{\alpha} \frac{q^{n}}{q-r_{h}} \eta_{h} .
$$

Proof. Note that

$$
\begin{aligned}
x_{n, \alpha}^{h, \delta}-x_{n, \alpha}^{\delta}= & x_{n-1, \alpha}^{h, \delta}-x_{n-1, \alpha}^{\delta}-\left[\left(P_{h} F^{\prime}\left(P_{h} x_{0}\right)+\alpha I\right)^{-1} P_{h}-\left(F^{\prime}\left(x_{0}\right)+\alpha I\right)^{-1}\right] \\
& \times\left(F\left(x_{n-1, \alpha}^{h, \delta}\right)-y^{\delta}+\alpha\left(x_{n-1, \alpha}^{h, \delta}-x_{0}\right)\right) \\
& -\left(F^{\prime}\left(x_{0}\right)+\alpha I\right)^{-1}\left[F\left(x_{n-1, \alpha}^{h, \delta}\right)-F\left(x_{n-1, \alpha}^{\delta}\right)+\alpha\left(x_{n-1, \alpha}^{h, \delta}-x_{n-1, \alpha}^{\delta}\right)\right] \\
= & \left(F^{\prime}\left(x_{0}\right)+\alpha I\right)^{-1}\left[F^{\prime}\left(x_{0}\right)\left(x_{n-1, \alpha}^{h, \delta}-x_{n-1, \alpha}^{\delta}\right)-\left(F\left(x_{n-1, \alpha}^{h, \delta}\right)-F\left(x_{n-1, \alpha}^{\delta}\right)\right)\right] \\
& -\left(F^{\prime}\left(x_{0}\right)+\alpha I\right)^{-1}\left[F^{\prime}\left(x_{0}\right) P_{h}-P_{h} F^{\prime}\left(P_{h} x_{0}\right) P_{h}\right]\left(P_{h} F^{\prime}\left(P_{h} x_{0}\right)+\alpha I\right)^{-1} \\
& \times P_{h}\left[\left(F\left(x_{n-1, \alpha}^{h, \delta}\right)-y^{\delta}+\alpha\left(x_{n-1, \alpha}^{h, \delta}-x_{0}\right)\right)\right] \\
= & \left(F^{\prime}\left(x_{0}\right)+\alpha I\right)^{-1}\left[F^{\prime}\left(x_{0}\right)\left(x_{n-1, \alpha}^{h, \delta}-x_{n-1, \alpha}^{\delta}\right)-\left(F\left(x_{n-1, \alpha}^{h, \delta}\right)-F\left(x_{n-1, \alpha}^{\delta}\right)\right)\right] \\
& -\left(F^{\prime}\left(x_{0}\right)+\alpha I\right)^{-1}\left[F^{\prime}\left(x_{0}\right)-P_{h} F^{\prime}\left(x_{0}\right)+P_{h} F^{\prime}\left(x_{0}\right)-P_{h} F^{\prime}\left(P_{h} x_{0}\right)\right]\left(x_{n, \alpha}^{h, \delta}-x_{n-1, \alpha}^{h, \delta}\right) \\
:= & \Gamma_{1}-\Gamma_{2},
\end{aligned}
$$

where

$$
\Gamma_{1}=\left(F^{\prime}\left(x_{0}\right)+\alpha I\right)^{-1}\left[F^{\prime}\left(x_{0}\right)\left(x_{n-1, \alpha}^{h, \delta}-x_{n-1, \alpha}^{\delta}\right)-\left(F\left(x_{n-1, \alpha}^{h, \delta}\right)-F\left(x_{n-1, \alpha}^{\delta}\right)\right)\right]
$$

and

$$
\Gamma_{2}=\left(F^{\prime}\left(x_{0}\right)+\alpha I\right)^{-1}\left[F^{\prime}\left(x_{0}\right)-P_{h} F^{\prime}\left(x_{0}\right)+P_{h} F^{\prime}\left(x_{0}\right)-P_{h} F^{\prime}\left(P_{h} x_{0}\right)\right]\left(x_{n, \alpha}^{h, \delta}-x_{n-1, \alpha}^{h, \delta}\right) .
$$


Using Lemma 2.2, one has

$$
\begin{aligned}
\left\|\Gamma_{1}\right\| \leq & l_{0} \int_{0}^{1} \| x_{0}-\left(x_{n-1, \alpha}^{h, \delta}+t\left(x_{n-1, \alpha}^{\delta}-x_{n-1, \alpha}^{h, \delta}\right)\|\| x_{n-1, \alpha}^{\delta}-x_{n-1, \alpha}^{h, \delta} \| d t\right. \\
\leq & l_{0} \int_{0}^{1}\left[t\left\|x_{0}-x_{n-1, \alpha}^{\delta}\right\|+(1-t)\left\|P_{h} x_{0}-x_{n-1, \alpha}^{h, \delta}\right\|\right. \\
& \left.+(1-t)\left\|P_{h} x_{0}-x_{0}\right\|\right]\left\|x_{n-1, \alpha}^{\delta}-x_{n-1, \alpha}^{h, \delta}\right\| d t \\
\leq & \frac{l_{0}}{2}\left[\frac{\eta}{1-r}+\frac{\eta_{h}}{1-r_{h}}+b_{h}\right]\left\|x_{n-1, \alpha}^{h, \delta}-x_{n-1, \alpha}^{\delta}\right\| \\
\leq & \frac{1}{2}\left[2 \tilde{\tilde{r}}+l_{0} b_{h}\right]\left\|x_{n-1, \alpha}^{h, \delta}-x_{n-1, \alpha}^{\delta}\right\| \\
\leq & q\left\|x_{n-1, \alpha}^{h, \delta}-x_{n-1, \alpha}^{\delta}\right\| .
\end{aligned}
$$

From Assumption 1.4, one has

$$
\begin{aligned}
\left\|\Gamma_{2}\right\|= & \left\|\left(F^{\prime}\left(x_{0}\right)+\alpha I\right)^{-1}\left[\left(I-P_{h}\right) F^{\prime}\left(x_{0}\right)-P_{h}\left(F^{\prime}\left(P_{h} x_{0}\right)-F^{\prime}\left(x_{0}\right)\right)\right]\left(x_{n, \alpha}^{h, \delta}-x_{n-1, \alpha}^{h, \delta}\right)\right\| \\
\leq & \left\|\left(F^{\prime}\left(x_{0}\right)+\alpha I\right)^{-1}\left(I-P_{h}\right) F^{\prime}\left(x_{0}\right)\right\| \\
& +\|\left(F^{\prime}\left(x_{0}\right)+\alpha I\right)^{-1} P_{h} F^{\prime}\left(x_{0}\right) \Phi\left(P_{h} x_{0}, x_{0}, x_{n, \alpha}^{h, \delta}-x_{n-1, \alpha}^{h, \delta}\right) \\
\leq & \frac{\Gamma_{h}+l_{0}\left\|F^{\prime}\left(x_{0}\right)\right\| b_{h}}{\alpha}\left\|x_{n, \alpha}^{h, \delta}-x_{n-1, \alpha}^{h, \delta}\right\| .
\end{aligned}
$$

It follows from (5.15), (5.16) and (5.17) that

$$
\begin{aligned}
\left\|x_{n, \alpha}^{h, \delta}-x_{n, \alpha}^{\delta}\right\| & \leq q\left\|x_{n-1, \alpha}^{h, \delta}-x_{n-1, \alpha}^{\delta}\right\|+\frac{\Gamma_{h}+l_{0}\left\|F^{\prime}\left(x_{0}\right)\right\| b_{h}}{\alpha}\left\|x_{n, \alpha}^{h, \delta}-x_{n-1, \alpha}^{h, \delta}\right\| \\
& \leq q^{n} b_{h}+\frac{\Gamma_{h}+l_{0}\left\|F^{\prime}\left(x_{0}\right)\right\| b_{h}}{\alpha} \eta_{h}\left(r_{h}^{n-1}+q r_{h}^{n-2}+\cdots+q^{n-1}\right) \\
& \leq q^{n} b_{h}+\frac{\Gamma_{h}+l_{0}\left\|F^{\prime}\left(x_{0}\right)\right\| b_{h}}{\alpha} \frac{q^{n}}{q-r_{h}} \eta_{h} .
\end{aligned}
$$

This completes the proof.

\section{ERror Bounds Under Source Conditions}

It follows from Proposition 3.1 and Theorem 3.1 that

$$
\left\|x_{\alpha}^{\delta}-x_{\alpha}\right\| \leq \frac{\delta}{\alpha}
$$

and

$$
\left\|x_{\alpha}-\hat{x}\right\| \leq\left(l_{0} r_{0}+1\right) \varphi(\alpha)
$$

where $x_{\alpha}$ is the unique solution of $F(x)+\alpha\left(x-x_{0}\right)=y$.

Combining the estimates in Theorem 2.1, Theorem 5.2, equation (6.1) and equation (6.2), we obtain the following Theorem.

Theorem 6.1. Let $x_{n, \alpha}^{h, \delta}$ be as in (5.8) and let the assumptions in Theorem 2.1 and Theorem 5.2 be satisfied. Then

$$
\left\|x_{n, \alpha}^{h, \delta}-\hat{x}\right\| \leq q^{n} b_{h}+\frac{\Gamma_{h}+l_{0}\left\|F^{\prime}\left(x_{0}\right)\right\| b_{h}}{\alpha} \frac{q^{n}}{q-r_{h}} \eta_{h}+\frac{r^{n} \eta}{1-r}+\frac{\delta}{\alpha}+\left(l_{0} r_{0}+1\right) \varphi(\alpha)
$$


Let

$$
n_{\delta}:=\min \left\{n: \max \left\{q^{n}, r^{n}\right\} \leq \delta\right\}
$$

and

$$
C_{m}:=\max \left\{b_{h}+\frac{\Gamma_{h}+l_{0}\left\|F^{\prime}\left(x_{0}\right)\right\| b_{h}}{q-r_{h}} \eta_{h}+\frac{\eta}{1-r}+1,\left(l_{0} r_{0}+1\right)\right\}
$$

Theorem 6.2. Let $x_{n, \alpha}^{h, \delta}$ be as in (5.8) and let the assumptions in Theorem 2.1 and Theorem 5.2 be satisfied. Let $n_{\delta}$ be as in (6.4) and let $C_{m}$ be as in (6.5). Then, for all $0<\alpha \leq 1$,

$$
\left\|x_{n_{\delta}, \alpha}^{h, \delta}-\hat{x}\right\| \leq C_{m}\left(\varphi(\alpha)+\frac{\delta}{\alpha}\right)
$$

6.1. A priori choice of the parameter. We observe that the error $\varphi(\alpha)+\frac{\delta}{\alpha}$ in (6.6) is of optimal order if $\alpha_{\delta}:=\alpha(\delta)$ satisfies $\alpha_{\delta} \varphi\left(\alpha_{\delta}\right)=\delta$. Using the function $\psi(\lambda):=\lambda \varphi^{-1}(\lambda), 0<\lambda \leq a$, we have $\delta=\alpha_{\delta} \varphi\left(\alpha_{\delta}\right)=\psi\left(\varphi\left(\alpha_{\delta}\right)\right)$. Hence, $\alpha_{\delta}=\varphi^{-1}\left(\psi^{-1}(\delta)\right)$. Using (6.6), we have the following result.

Theorem 6.3. Let $\psi(\lambda):=\lambda \varphi^{-1}(\lambda)$ for $0<\lambda \leq a$, and assumptions in Theorem 6.2 holds. For $\delta>0$, let $\alpha=: \alpha_{\delta}=\varphi^{-1}\left(\psi^{-1}(\delta)\right)$. Let $n_{\delta}$ be as in (6.4). Then $\left\|x_{n_{\delta}, \alpha}^{h, \delta}-\hat{x}\right\|=O\left(\psi^{-1}(\delta)\right)$.

6.2. An adaptive choice of the parameter. We will present a parameter choice rule based on the adaptive method studied in $[14,16]$. The regularization parameter $\alpha$ is selected from the finite set

$$
D_{M}(\alpha):=\left\{\alpha_{i}=\mu^{i} \alpha_{0}, i=0,1, \cdots, M\right\},
$$

where $\mu>1$ and $M$ is such that $\alpha_{M}<1 \leq \alpha_{M+1}$. We choose $\alpha_{0}:=\sqrt{\delta}$ because in general $\varphi(\lambda)=$ $\lambda^{v}, 0<v \leq 1$ and in this case the best possible error estimate is order $\bigcirc(\sqrt{\delta})$. From Theorem 6.3, it follows that such an accuracy cannot be guaranteed for $\alpha<\sqrt{\delta}$. Let

$$
n_{M}:=\min \left\{n: \max \left\{q^{n}, r^{n}\right\} \leq \delta\right\}
$$

and $x_{i}:=x_{n_{M}, \alpha_{i}}^{h, \delta}$. We select $\alpha=\alpha_{i}$ from $D_{M}(\alpha)$ and operates only with corresponding $x_{i}, i=0,1, \cdots, M$.

Theorem 6.4. (cf. Theorem 3.5) Assume that there exists $i \in\{0,1,2, \cdots, M\}$ such that $\varphi\left(\alpha_{i}\right) \leq \frac{\delta}{\alpha_{i}}$. Let assumptions of Theorem 6.2 and Theorem 6.3 hold and let $l:=\max \left\{i: \varphi\left(\alpha_{i}\right) \leq \frac{\delta}{\alpha_{i}}\right\}<M$,

$$
k:=\max \left\{i:\left\|x_{i}-x_{j}\right\| \leq 4 C_{m} \frac{\delta}{\alpha_{j}}, j=0,1,2, \cdots, i\right\} .
$$

Then $l \leq k$ and $\left\|\hat{x}-x_{k}\right\| \leq c \psi^{-1}(\delta)$, where $c=6 C_{m} \mu$.

\section{IMPLEMENTATION OF THE ADAPTIVE CHOICE RULE}

In this section, we provide an algorithm for the determination of a parameter fulfilling the balancing principle (6.9) and provide a starting point for iteration (5.8) to approximate the unique solution $x_{\alpha}^{\delta}$ of (1.3). The choice of the starting point involves the following steps:

- Choose $\alpha_{0}=\sqrt{\delta}, \mu>1$ and $q<1$.

- Choose $x_{0} \in D(F)$ such that $\left\|x_{0}-\hat{x}\right\| \leq \rho$ and $\eta_{h}$ satisfying (5.9).

The choice of the stopping index $n_{M}$ involves the following step:

- Choose $n_{M}$ such that $n_{M}=\min \left\{n: \max \left\{q^{n}, r^{n}\right\} \leq \delta\right\}$.

Finally the adaptive algorithm associated with the choice of the parameter specified in Theorem 6.4 involves the following steps. 


\subsection{Algorithm.}

- Set $i \leftarrow 0$.

- Solve $x_{i}:=x_{n_{M}, \alpha_{i}}^{h, \delta}$ via iteration (5.8).

- If $\left\|x_{i}-x_{j}\right\|>4 C_{m} \frac{\sqrt{\delta}}{\mu^{j}}, \quad j \leq i$, then we take $k=i-1$.

- Set $i=i+1$ and return to step 2 .

\section{EXAMPLES}

In this section, we consider some simple examples satisfying the assumptions made in this paper and presents a few of examples.

We consider the operator $F: L^{2}[0,1] \rightarrow L^{2}[0,1]$ defined by [15, Example 6.1]

$$
F(x)(s)=K^{*} K(x)(s)+f(s), \quad x, f \in L^{2}[0,1], s \in[0,1],
$$

where $K: L^{2}[0,1] \rightarrow L^{2}[0,1]$ is a compact linear operator such that the range of $K$ denoted by $R(K)$ is not closed in $L^{2}[0,1]$. Then the equation $F(x)=y$ is ill-posed as $K$ is compact with non-closed range. The Fréchet derivative $F^{\prime}($.$) of F$ is given by

$$
F^{\prime}(x) z=K^{*} K z, \quad \forall x, z \in L^{2}[0,1] .
$$

So, $F$ is monotone on $L^{2}[0,1]$. Further, for $x, y, z \in L^{2}[0,1]$, one has

$$
\left[F^{\prime}(x)-F^{\prime}(y)\right] z=0 \text {. }
$$

It is obvious that Assumption 1.4 holds. Since $\Phi(x, y, z)=0 \leq l_{0}\|z\|\|x-y\|, \forall l_{0} \geq 0$ we can choose $\eta_{h}$ large enough in step 2 of the algorithm. Further, due to (8.2), $x_{m+1, \alpha}^{h, \delta}$ only needs one step to compute. This can be seen as follows:

$$
x_{m+1, \alpha}^{h, \delta}=x_{m, \alpha}^{h, \delta}-\left(P_{h} F^{\prime}\left(P_{h} x_{0}\right)+\alpha I\right)^{-1} P_{h}\left[F\left(x_{m, \alpha}^{h, \delta}\right)-y^{\delta}+\alpha\left(x_{m, \alpha}^{h, \delta}-x_{0}\right)\right],
$$

i.e.,

$$
\begin{aligned}
\left(P_{h} F^{\prime}\left(P_{h} x_{0}\right)+\alpha I\right) P_{h} x_{m+1, \alpha}^{h, \delta} & =\left(P_{h} F^{\prime}\left(P_{h} x_{0}\right)+\alpha I\right) P_{h} x_{m, \alpha}^{h, \delta}-P_{h}\left[F\left(x_{m, \alpha}^{h, \delta}\right)-y^{\delta}+\alpha\left(x_{m, \alpha}^{h, \delta}-x_{0}\right)\right] \\
& =\left(P_{h} K^{*} K+\alpha I\right) P_{h} x_{m, \alpha}^{h, \delta}-P_{h}\left[K^{*} K x_{m, \alpha}^{h, \delta}+f-y^{\delta}+\alpha\left(x_{m, \alpha}^{h, \delta}-x_{0}\right)\right] \\
& =-P_{h}\left(f-y^{\delta}-\alpha x_{0}\right) .
\end{aligned}
$$

Next, we give the details for implementing the algorithm given in the above section. Let $\left(V_{n}\right)$ be a sequence of finite dimensional subspaces of $X$ and let $P_{h}, h=1 / n$ denote the orthogonal projection on $X$ with range $R\left(P_{h}\right)=V_{n}$. We assume that $\operatorname{dim}_{n}=n+1$, and $\left\|P_{h} x-x\right\| \rightarrow 0$ as $h \rightarrow 0$ for all $x \in X$. Let $\left\{v_{1}, v_{2}, \cdots, v_{n+1}\right\}$ be a basis of $V_{n}, n=1,2, \cdots$. Note that $x_{m+1, \alpha}^{h, \delta} \in V_{n}$. Thus, $x_{m+1, \alpha}^{h, \delta}$ is of the form $\sum_{i=1}^{n+1} \lambda_{i} v_{i}$ for some scalars $\lambda_{1}, \lambda_{2}, \cdots, \lambda_{n+1}$. It can be seen that $x_{m+1, \alpha}^{h, \delta}$ is a solution of (8.4) if and only if $\bar{\lambda}=\left(\lambda_{1}, \lambda_{2}, \cdots, \lambda_{n+1}\right)^{T}$ is the unique solution of

$$
\left(M_{n}+\alpha B_{n}\right) \bar{\lambda}=\bar{a}
$$

where $M_{n}=\left(\left\langle K v_{i}, K v_{j}\right\rangle\right), i, j=1,2, \cdots, n+1 B_{n}=\left(\left\langle v_{i}, v_{j}\right\rangle\right), i, j=1,2, \cdots, n+1$ and

$$
\bar{a}=\left(\left\langle P_{h}\left(y^{\delta}+\alpha x_{0}-f\right), v_{i}\right\rangle\right)^{T}, i=1,2, \cdots, n+1 .
$$

Note that (8.5) is uniquely solvable because $M_{n}$ is a positive definite matrix (i.e., $x M_{n} x^{T}>0$ for all non-zero vector $\mathrm{x}$ ) and $B_{n}$ is an invertible matrix. 
8.1. Numerical Examples. In order to illustrate the method considered in the above section, we consider the space $X=Y=L^{2}[0,1]$ and $K: L^{2}[0,1] \rightarrow L^{2}[0,1]$ as the Fredholm integral operator

$$
K(x)(s)=\int_{0}^{1} k(s, t) x(t) d t,
$$

with

$$
k(t, s)= \begin{cases}0, & t \leq s \\ t-s, & t>s .\end{cases}
$$

We apply the Algorithm in Section 7 by choosing $V_{n}$ as the space of linear splines in a uniform grid of $n+1$ points in $[0,1]$. Specifically, for fixed $n$, we consider $t_{i}=\frac{i-1}{n}, i=1,2, \cdots, n+1$ as the grid points. We take the basis function $v_{i}, i=1,2, \cdots, n+1$ of $V_{n}$ as follows:

$$
v_{1}(t)= \begin{cases}\frac{t_{2}-t}{t_{2}}, & 0=t_{1} \leq t \leq t_{2} \\ 0, & t_{2} \leq t \leq t_{n+1}=1\end{cases}
$$

for $j=2,3, \cdots, n$,

$$
v_{j}(t)= \begin{cases}0, & 0=t_{1} \leq t \leq t_{j-1}, \\ \frac{t-t_{j-1}}{t_{j}-t_{j-1}}, & t_{j-1} \leq t \leq t_{j}, \\ \frac{t_{j+1}-t}{t_{j+1}-t_{j}}, & t_{j} \leq t \leq t_{j+1} \\ 0, & t_{j+1} \leq t \leq t_{n+1}=1\end{cases}
$$

and

$$
v_{n+1}(t)= \begin{cases}0, & 0 \leq t \leq t_{n} \\ \frac{t-t_{n}}{t_{n+1}-t_{n}}, & t_{n} \leq t \leq t_{n+1}\end{cases}
$$

Let $P_{h}$ be the orthogonal projection onto $V_{n}$. We note that, for $x \in C[0,1]$,

$$
\begin{aligned}
\left\|P_{h} x-x\right\|_{2} & =\operatorname{dist}\left(x, R\left(P_{h}\right)\right) \\
& \leq\left\|\pi_{n} x-x\right\|_{2} \\
& \leq\left\|\pi_{n} x-x\right\|_{\infty}
\end{aligned}
$$

where $\pi_{n}$ is the (piecewise linear) interpolatory projection onto $V_{n}$. It is known that $\left\|\pi_{n} x-x\right\|_{\infty} \rightarrow 0$ as $n \rightarrow \infty$. Therefore using the fact that $C[0,1]$ is dense in $L^{2}[0,1]$, it follows that $\left\|P_{h} x-x\right\|_{2} \rightarrow 0$ for all $x \in L^{2}[0,1]$. The elements $K v_{i}, i=1,2, \cdots, n+1$, the entries of the matrix $B_{n}, M_{n}$ and $\bar{a}$ are computed explicitly. For the operator $K$ defined by (8.6) and (8.7), $\Gamma_{h}=\gamma_{h}=\left\|\left(I-P_{h}\right) F^{\prime}\left(x_{0}\right)\right\|=\left\|\left(I-P_{h}\right) K^{*} K\right\|=$ $O\left(n^{-2}\right)$ (see, [11]).

Example 8.1. Take $y=\frac{1}{720}\left(26+s^{6}-6 s^{5}+15 s^{4}-36 s\right)+f(s)$, where $f(s)=s^{2}$ and $x_{0}=0$. Then the exact solution is $\hat{x}=\frac{1}{2}(s-1)^{2}$. Since $\hat{x}-x_{0}=\hat{x}=K^{*} 1 \in R\left(K^{*}\right)=R\left(F^{\prime}(\hat{x})^{1 / 2}\right), \varphi(\lambda)=\lambda^{1 / 2}$. Hence $\psi^{-1}(\delta)=\varphi\left(\alpha_{\delta}\right)=(\delta)^{1 / 3}$. This implies that $\left\|\hat{x}-x_{k}\right\| \leq c \psi^{-1}(\delta)$, where $c=6 C_{m} \mu$. The result are given in the following Table and Figures. 


\begin{tabular}{|c|c|c|c|}
\hline $\mathrm{n}$ & $\mathrm{k}$ & $e_{k}$ & $\frac{e_{k}}{\psi^{-1}(\delta)}$ \\
\hline 4 & 100 & 0.0377 & 0.1385 \\
\hline 8 & 99 & 0.0385 & 0.1400 \\
\hline 16 & 99 & 0.0385 & 0.1399 \\
\hline 32 & 99 & 0.0385 & 0.1400 \\
\hline 64 & 99 & 0.0385 & 0.1400 \\
\hline 128 & 99 & 0.0385 & 0.1400 \\
\hline 256 & 99 & 0.0385 & 0.1400 \\
\hline 512 & 99 & 0.0385 & 0.1400 \\
\hline 1024 & 99 & 0.0385 & 0.1400 \\
\hline
\end{tabular}

Table 3.1: $\delta=0.001 ; \mu=1.002$.

Here $e_{k}:=\left\|x_{k}-\hat{x}\right\|$ and $y^{\delta}=y+\delta$.

Remark 8.1. The last column of the Table shows that $e_{k}=O\left(\psi^{-1}(\delta)\right)$. From computation, we observe that due to the round off error $k$ and $e_{k}$ remains as a constant for large values of $n$.



$\mathrm{n}=8$

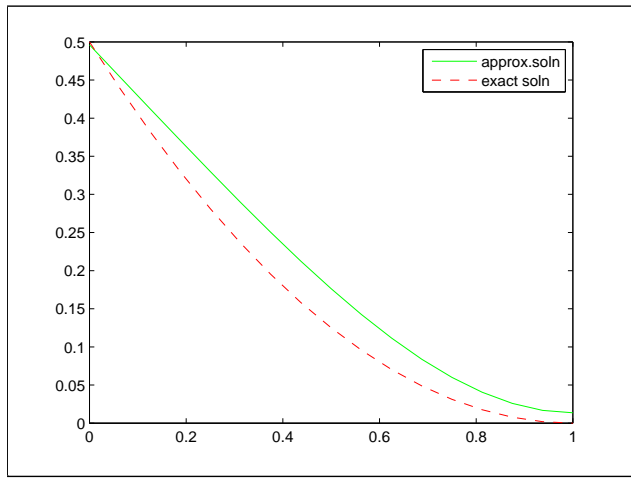

$\mathrm{n}=32$

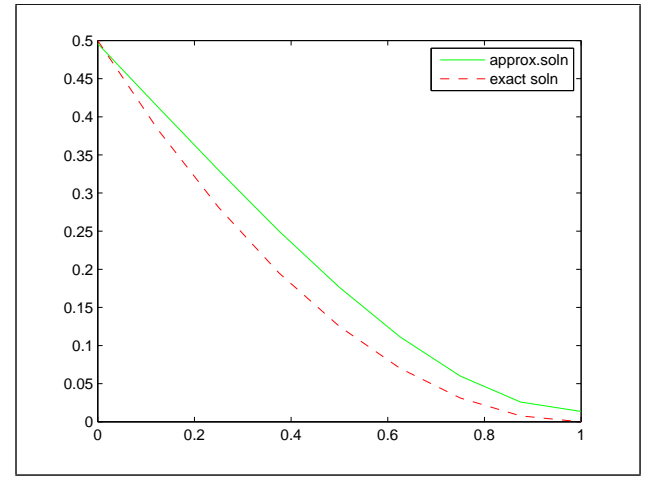

$\mathrm{n}=16$

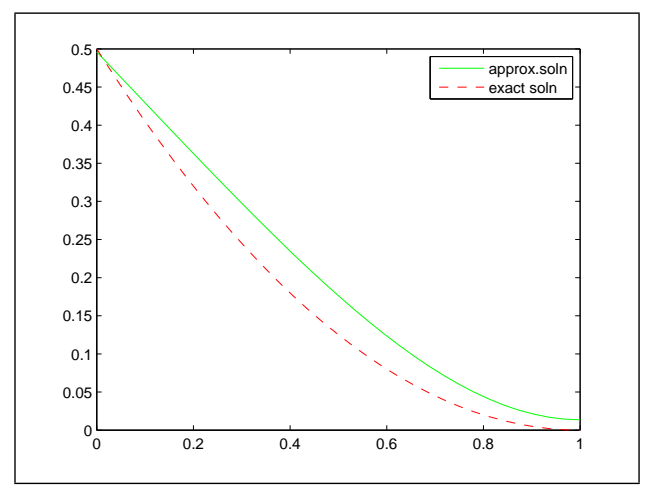

$\mathrm{n}=64$

FIGURE 1. Curve of the exact and approximate solutions 


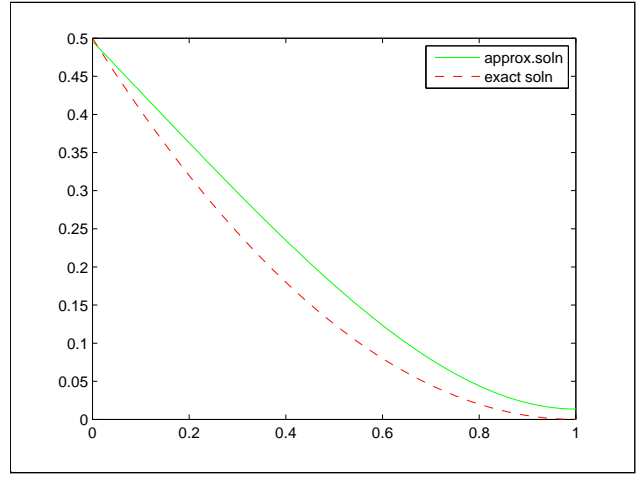

$\mathrm{n}=128$

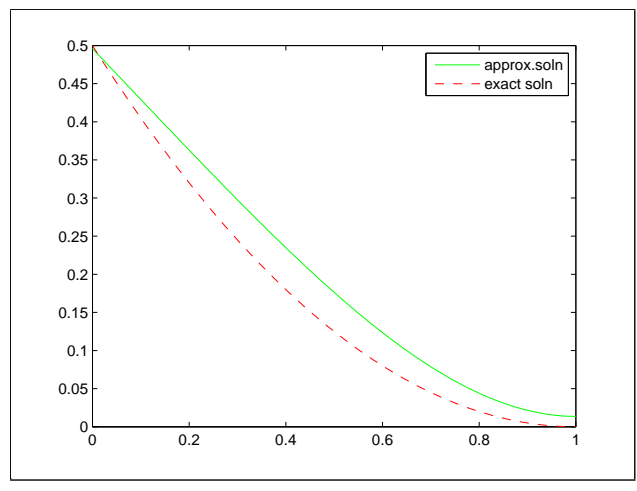

$\mathrm{n}=512$

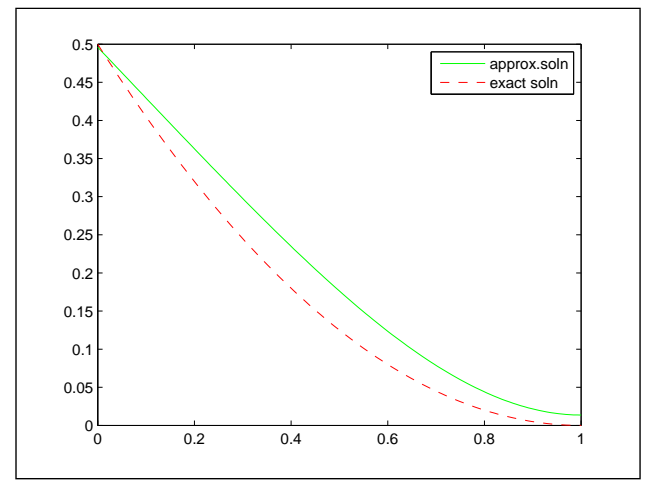

$\mathrm{n}=256$

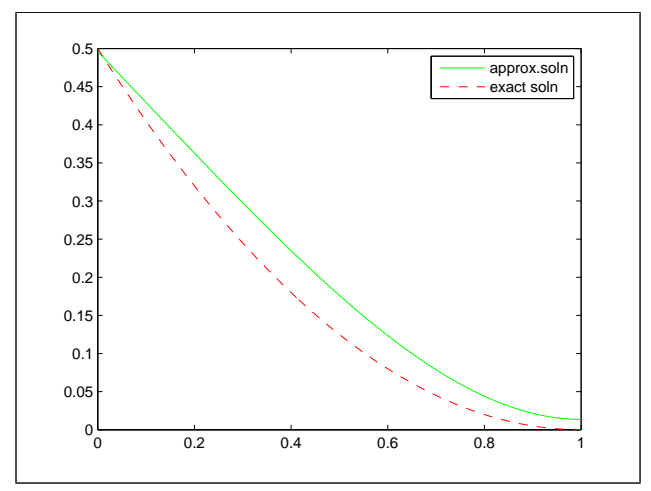

$\mathrm{n}=1024$

FIGURE 2. Curve of the exact and approximate solutions

\section{Concluding Remark}

In this paper, we considered an iteratively regularized projection method for solving the nonlinear ill-posed operator equation $F(x)=y$, when the available data is $y^{\delta}$ in place of the exact data $y$ with $\left\|y-y^{\delta}\right\| \leq \delta$. It is assumed that $F$ is Fréchet differentiable in a neighborhood of some initial guess $x_{0}$ of the actual solution $\hat{x}$. The procedure involves finding the fixed point of the function $G_{h}(x):=$ $x-\left(P_{h} F^{\prime}\left(P_{h} x_{0}\right)+\alpha I\right)^{-1} P_{h}\left(F(x)-y^{\delta}+\alpha\left(x-x_{0}\right)\right)$, in a finite dimensional subspace $X_{h}$ of $X$ iteratively, where $P_{h}$ is the orthogonal projection on to $X_{h}$. For choosing the regularization parameter $\alpha$, we employed the adaptive method suggested by Pereversev and Schock in [16] and the stopping rule is based on a majorizing sequence. Our numerical experiments show that if $\alpha$ is chosen according to the balancing principle (6.9), then $\left\|x_{k}-\hat{x}\right\| \leq c \psi^{-1}(\delta)$.

\section{REFERENCES}

[1] I.K. Argyros, Convergence and Application of Newton-type Iterations, Springer, New York, 2008.

[2] I.K.Argyros, Approximating solutions of equations using Newton's method with a modified Newton's method iterate as a starting point, Rev. Anal. Numer. Theor. Approx. 36, (2007), 123-138.

[3] I .K. Argyros, A semilocal convergence for directional Newton methods, Math. Comput. 80 (2011), 327-343.

[4] I.K. Argyros, S. Hilout, Weaker conditions for the convergence of Newton's method, J. complexity, 28 (2012), $364-387$.

[5] I.K. Argyros, Y.J. Cho, S.Hilout, Numerical methods for equations and its applications, CRC Press, New York, 2012. 
[6] A. Bakushinsky, A. Smirnova, On application of generalized discrepancy principle to iterative methods for nonlinear ill-posed problems, Numer. Funct. Anal. Optim. 26 (2005), 35-48.

[7] I.K. Argyros, Santhosh George, On the convergence of Broyden's method with regularity continuous divided differences and restricted convergence domains, J. Nonlinear Funct. Anal. 2017 (2017), Article ID 21.

[8] P. Deuflhard, H.W. Engl, O. Scherzer, A convergence analysis of iterative methods for the solution of nonlinear ill-posed problems under affinely invariant conditions, Inverse Probl. 14 (1998), 1081-1106.

[9] S. George, A. I.Elmahdy, An analysis of Lavrentiev regularization for nonlinear ill-posed problems using an iterative regularization method, Int. J. Comput. Appl. Math. 5, (2010), 369-381.

[10] R. Gorenflo, B. Hofmann, On autoconvolution and regularization, Inverse Probl. 10 (1994), 353-373.

[11] C.W. Groetsch, J.T. King, D. Murio, Asymptotic analysis of a finite element method for Fredholm equations of the first kind, In: Treatment of Integral Equations by Numerical Methods, Edited by: C. T. Baker and G. F. Miller, pp. 1-11, Academic Press, London, 1982.

[12] M. Hanke, A. Neubauer, O. Scherzer, A convergence analysis of the Landweber iteration for nonlinear ill-posed problems, Numer. Math. 72 (1995), 21-37.

[13] P. Mahale, M.T. Nair, Iterated Lavrentiev regularization for nonlinear ill-posed problems, ANZIAM J. 51 (2009), $191-217$.

[14] P. Mathe, S.V. Perverzev, Geometry of linear ill-posed problems in variable Hilbert scales, Inverse Probl. 19 (2003), 789-803.

[15] M.T. Nair, P. Ravishankar, Regularized versions of continuous Newton's method and continuous modified Newton's method under general source conditions, Numer. Funct. Anal. Optim. 29 (2008), 1140-1165.

[16] S. V. Perverzev, E. Schock, On the adaptive selection of the parameter in regularization of ill-posed problems, SIAM J. Numer. Anal. 43 (2005), 2060-2076.

[17] J. Qi-Nian, Error estimates of some Newton-type methods for solving nonlinear inverse problems in Hilbert scales, Inverse Probl. 16 (2000), 187-197.

[18] J. Qi-Nian, On the iteratively regularized Gauss-Newton method for solving nonlinear ill-posed problems, Math. Comput. 69 (2000), 1603-1623.

[19] A.G. Ramm, Inverse problems: Mathematical and Analytical Techniques with Applications to Engineering, Springer, 2005.

[20] U. Tautanhahn, On the method of Lavrentiev regularization for nonlinear ill-posed problems, Inverse Probl. 18 (2002), 191-207. 